\title{
Investigating the Infrared Properties of Candidate Blazars
}

\author{
Jessica Hall \\ Office of Science, Science Undergraduate Laboratory Internship Program (SULI) \\ University of Southern California \\ Stanford Linear Accelerator Center \\ Menlo Park, California \\ August 18, 2005
}

Prepared in partial fulfillment of the requirements of the Office of Science, U.S.

Department of Energy Science Undergraduate Laboratory Internship (SULI) Program under the direction of Grzegorz Madejski and Seth Digel in the Gamma-ray Large-Area Space Telescope (GLAST) division at Stanford Linear Accelerator Center.

Participant:

Signature

Research Advisors:

Signature

Signature 


\section{Table of Contents}

$\underline{\text { Page }}$

$\begin{array}{lll}\text { Abstract } & \text { iii }\end{array}$

Introduction 1

Methods 3

$\begin{array}{ll}\text { Results } & 6\end{array}$

$\begin{array}{ll}\text { Discussion and Conclusions } & 7\end{array}$

$\begin{array}{ll}\text { Acknowledgments } & 11\end{array}$

$\begin{array}{ll}\text { Works Cited } & 12\end{array}$

$\begin{array}{ll}\text { Other References } & 12\end{array}$

$\begin{array}{ll}\text { Tables } & 14\end{array}$

$\begin{array}{ll}\text { Figures } & 16\end{array}$ 


\begin{abstract}
Investigating the Infrared Properties of Candidate Blazars. JESSICA HALL (University of Southern California, LA, 90007) GRZEGORZ MADEJSKI (Stanford Linear Accelerator Center, Menlo Park, CA 94025) SETH DIGEL (Stanford Linear Accelerator Center, Menlo Park, CA 94025).
\end{abstract}

Blazars are active galaxies with super-massive black holes, containing jets that accelerate plasma material and produce radiation. They are unique among other active galaxies for properties such as rapid variability and the lack of emission lines. The double-peaked spectral energy distribution (SED) found for most blazar objects suggests that synchrotron radiation and Compton scattering occurs in the jets. This study is an investigation of the infrared (IR) spectra of a selected population of blazar candidates, focusing on the IR properties of objects within the three types of blazars currently recognized by their spectral characteristics at other wavelengths. Using blazar candidates found in a recent study of the northern sky (Sowards-Emmerd et al., The Astrophysical Journal, 2005), IRAS data for 12, 25, 60, and $100 \mu \mathrm{m}$, as well as any available data from 2MASS and EGRET, were located. The synchrotron peak of the SED of each object was expected to occur anywhere in the infrared (IR) to soft X-ray range. However, peaks were generally found to lie in the IR range, suggesting potential selection biases. An analysis of selection techniques reveals that the figure of merit used in the original survey is engineered to select objects with a Compton scattering peak luminosity occurring in the $\mathrm{GeV}$ range, the energy band most easily detected by the upcoming GLAST mission. Therefore, this figure of merit selection process should be used to compile a list of blazar candidates for further study in anticipation of the launch of the satellite. 


\section{INTRODUCTION}

Blazars are active galaxies with super-massive black holes at their cores. The common understanding is that the black holes are rotating and have accretion disks. The physics of this structure often causes jets of high-energy particles that accelerate plasma material out from the nucleus. Compton scattering, and perhaps other processes, creates gamma rays in these jets. One distinguishing characteristic of blazars relative to other active galaxies is that their jets are more or less aligned with the line of sight to the Earth. Relativistic beaming boosts the energy of $\gamma$-rays along the jets, making blazars extremely bright sources of high-energy $\gamma$-rays. Blazars are unusual among other active galaxies for properties such as rapid variability and the lack of emission lines. They are defined by a flat featureless spectrum, running from the radio range often into the optical, with a strong non-thermal optical component.

Recent research into the methods of identifying blazar candidates has focused on correlation of radio, $\mathrm{X}$-ray, and $\gamma$-ray data from different astronomical catalogs to view different characteristics of these objects. Ideally, the data included in such a comparison would be contemporaneous; the variability of blazars makes it difficult to compare properties without obtaining data at all wavelengths at the same time. However, a multiwavelength data compilation for an object can be useful to analyze the general characteristics of a population of blazar candidates, even if the data are not taken simultaneously.

The unique double-peaked spectral energy distribution (SED) usually observed in multi-wavelength correlations of blazars, typically falling in the infrared (IR) to soft xray range for one peak and $\gamma$-ray bands for the other peak, suggests that synchrotron 
radiation and inverse Compton scattering of electrons occurs in the jets. Based on their SEDs, blazars have been classified into 3 groups: High-energy peaked BL Lacs (HBL), low-energy peaked BL Lacs (LBL), and flat-spectrum radio quasars (FSRQ). Blazars had already been divided into BL Lacs and FSRQ due to certain properties they exhibit, but recent observations have shown that further division was necessary. According to Ghissellini et al. in their unifying scheme for blazars:

'(i) HBL are sources characterized by the lowest intrinsic power and the weakest external radiation field (no or weak emission lines). Consequently the cooling is less dramatic and particles can be present with energies high enough to produce synchrotron emission extending to soft $\mathrm{X}$-ray energies and $\mathrm{TeV}$ radiation through the [synchrotron self-Compton] SSC process. [T] he inverse Compton cooling [being] ineffective, the Compton dominance is expected to be small;

(ii) LBL are intrinsically more powerful than HBL and in some cases the external field can be responsible for most of the cooling. The stronger cooling limits the particle energy implying that the synchrotron and inverse Compton emission peak at lower frequencies, in the optical and $\mathrm{GeV}$ bands, respectively, with a larger Compton dominance parameter;

(iii) FSRQ represent the most powerful blazars, where the contribution from the external radiation to the cooling is the greatest. The emission by synchrotron and [external Compton] EC cannot extend [to] frequencies larger than the IR and $\mathrm{MeV}-\mathrm{GeV}$ bands and the $\gamma$-ray radiation completely dominates the radiative output. Within this class, there is a hint of a further sub-division between low and high polarization objects, with a tendency for LPQ to be more extreme (lower values of $\gamma_{\text {peak }} . .$. and so on).' (1: 14)

This study is a follow-up investigation of the IR properties of a blazar candidate

population, in hopes of identifying the nature of the double-peaked SED structure in more detail. Other multi-wavelength studies of blazars have been made in the past, with objects selected for their radio, X-ray, or $\gamma$-ray properties. However, IR data have been scarce in these surveys, as has sufficient $\gamma$-ray coverage of the sky. The limitations of these data will be discussed in the next section. This project focused on expanding on a database already containing data for many wavelengths, provided by Sowards-Emmerd et al. (2). The merit of such a study is obvious; blazars may be very bright in the $\gamma$-ray band, but the 
limited positional resolution of the former EGRET mission makes a $\gamma$-ray selected population impossible to obtain with any accuracy. (Positions of EGRET sources are typically uncertain by 90' or more and the great majority of EGRET sources were still unidentified after the mission.) The Gamma-ray Large Area Space Telescope (GLAST), scheduled to launch in 2007, is expected to detect more than an order of magnitude greater numbers of blazars, and will cover the entire sky approximately every 3 hours. This instrument will greatly improve the study of AGN in general, but until it is in operation, studies of data available in other energy bands are useful to select objects likely to be blazars that GLAST will detect. For this reason, the analysis herein includes any IR and near-IR data available for the objects in question.

\section{METHODS}

A blazar candidate population from a recent study of the northern sky by Sowards-Emmerd et al. (2), selected on a figure of merit (FoM) basis to be similar in property to those blazars that had shown up in the EGRET surveys, was used for analysis. Table 1 of that survey (2:96) was the source database for this study, and included any available radio, X-ray, and red wavelength data for the objects selected by their method. While this method is outlined more in detail within that paper, the definition of FoM they used is as follows (2:96):

FoM $=n_{8.4 G H z} * n_{\alpha} * n_{X-\text { ray }} *$ positional dependence [based on location of radio source], where these functions are defined as

$n_{8.4 \mathrm{GHz}}=-3.47+2.45 \log s_{8.4}-0.34\left(\log s_{8.4}\right)^{2}$,

$n_{\alpha}=\operatorname{median}\left(0,0.19-0.35 \alpha_{8.4 / 1.4}, 0.4\right)$,

$n_{X-\text { ray }}=0.5+$ median $[0,0.49+0.41 \log ($ counts $/ \mathrm{s}), 0.5]$

The definition of the figure of merit above made use of the radio flux at $8.4 \mathrm{GHz}$, as found by the Cosmic Lens All-Sky Survey (CLASS), as well as the radio spectral index. 
The x-ray flux was also used, in units of counts per second, and was obtained from the ROSAT All-Sky Survey (RASS). Each of the n terms described above were calibrated to fall in the range of 0 to 1 , defining the FoM as a product probability that would fall in the range of 0 to 1 as well. Because they were searching for EGRET-like sources in this study, instead of matching radio sources to EGRET sources as they had done in a 2003 survey, the positional dependence of the radio source was omitted in their general method, and will not be included further in this survey. Once these objects were chosen by the Sowards-Emmerd et al. figure of merit, the group used different methods, such as archival data, to identify the selected targets as blazar candidates or other phenomena. That study returned a database of over 700 objects, only a portion of which were blazar candidates.

For the purposes of this study, only the objects identified as BL Lac and FSRQ in that survey were considered for infrared analysis. The other objects identified in the northern sky survey were often not considered active galaxies, and many were unidentified, rendering them useless for this study. After obtaining the original database, condensing it to the $\sim 500$ objects of BL Lac or FSRQ type and converting all flux values to the Jansky unit, the NASA/IPAC Extragalactic Database (NED) was used to fill in the rest of the broadband data. The Infrared Astronomical Satellite (IRAS) data for 12, 25, 60, and $100 \mu \mathrm{m}$ wavelengths, as well as near-IR data available from the 2-Micron AllSky Survey (2MASS) in the J, $\mathrm{H}$, and $\mathrm{K}_{\mathrm{s}}$ bands $(1.24,1.66$, and $2.16 \mu \mathrm{m}$, respectively) were used in this study. EGRET data were also found for the objects that showed up in the IR or near-IR ranges. 
After obtaining all available data in the various wavelength bands, a $v$ vs. $v \mathrm{~F}_{v}$ graph was created to show the spectral energy distribution (SED) of each source (Figure 1). The frequency values used for each wavelength band are included in Table 1. An algorithm was used to find the effective average frequency $<v>$ in the $\gamma$-ray band during the conversion of photon count data from EGRET to Janskys, based on the spectral index of the EGRET source. The range of the EGRET flux values for each source was plotted at this average frequency on the SED graph.

Only the graphs with sufficient IR or near-IR data, or a nearly complete SED, were considered for curve-fit analysis. The SEDs of these objects, shown in a log-log scale, had a parabola curve fitted to them (also in log-log scale) to evaluate where the peak synchrotron radiation occurred for the non-simultaneous data. The locations of the synchrotron peak frequencies were noted and used for further analysis.

To see if there was a correlation between the FoM defined in the original data set and the location of the synchrotron peak frequencies found by the graphing program for each object, a $v_{\text {peak }}$ vs. FoM graph was created (Figure 2). The reason why this graph was not considered as sufficient to reveal correlation effects is explained in the following discussion. Because of this, a new graph was created, scaling the FoM for each object by its defined redshift (Figure 3). The FoM was defined using spectral indexes and flux values. To correct for redshift, one must consider the FoM in terms of distance, changing its focus from flux to absolute luminosity. The luminosity distance to be used here can be defined as follows (3:4):

$D_{L}=\frac{c}{H_{0}}(1+z)^{2} \int_{0}^{z} \frac{d z^{\prime}}{E\left(z^{\prime}\right)} \quad$ where $\quad E(z)=\sqrt{\Omega_{M}(1+z)^{3}+\Omega_{K}(1+z)^{2}+\Omega_{\Lambda}}$ 
All values for $\mathrm{D}_{\mathrm{L}}$ for the 18 objects that had a defined redshift are included in Table 2 .

Here the values of $\Omega_{\mathrm{M}}=0.3, \Omega_{\Lambda}=0.7$, and $\mathrm{H}_{0}=70 \mathrm{~km} / \mathrm{s} / \mathrm{Mpc}$ were used. $\Omega_{\mathrm{K}}$ is

considered negligible here. To change flux values to luminosity:

$$
\begin{aligned}
& L=\frac{F^{*} 4 \pi * D_{L}^{2}}{1+z} \\
& L_{\text {ratio }}=F_{\text {ratio }} * \frac{\left(4 \pi * D_{L}^{2}\right) /(1+z)}{\left(4 \pi * D_{\text {med }}^{2}\right) /(1+z)}=F_{\text {ratio }} *\left(\frac{D_{L}}{D_{\text {med }}}\right)^{2} \text { where } D_{\text {med }} \text { is the median of } D_{L} \text { for all objects }
\end{aligned}
$$

Thus, the modified FoM can be defined as:

scaled FoM $=n_{8.4 \mathrm{GHz}} * n_{\alpha} * n_{X-\mathrm{ray}}$,

where these functions are defined as

$n_{8.4 \mathrm{GHz}}=-3.47+2.45 \log \left(s_{8.4} *\left(D / D_{\text {med }}\right)^{2}\right)-0.34\left(\log \left(s_{8.4} *\left(D / D_{\text {med }}\right)^{2}\right)\right)^{2}$,

$n_{\alpha}=$ median $\left(0,0.19-0.35 \alpha_{8.4 / 1.4}, 0.4\right)$,

$n_{X-\text { ray }}=0.5+$ median $\left[0,0.49+0.41 \log \left((\right.\right.$ count $\left.\left./ s) *\left(D / D_{\text {med }}\right)^{2}\right), 0.5\right]$

This modified FoM is scaled in terms of luminosity instead of flux values, and is still relatable to the peak frequency. Here the original constants chosen by the SowardsEmmerd et al. study have not been changed. While this has resulted in $\mathrm{n}$ terms that are no longer scaled to fall between 0 and 1 , the modified product probability figure of merit is still useful for comparison. The peak frequencies for each object as well as the changed FoM are included in Table 2 for reference. The scaled FoM graph (Figure 3) was more useful to this investigation, and will be discussed further below.

\section{RESULTS}

In the course of searching databases, it became clear that very limited infrared data exist for the objects in this sample. This made the number of objects to be analyzed much smaller than the original sample. About 22 objects out of the original 504 BL Lac and FSRQ sources considered from the survey had adequate data in almost all 
wavelengths, only 20 of which had a distinctive synchrotron peak frequency found by the computer curve-fitting procedure. The frequency coverage and sensitivity limit of each instrument used for the data necessary to this project are included in Table 3. Several factors could have brought about the outcome of so little data in the IR and $\gamma$-ray bands. The positional accuracy of the source detections for various instruments could have placed the matching object out of the search radius used (about 2 arc minutes). Also, some objects may not have been detected because their cycle of variability was at a low when that portion of the sky was scanned by the telescopes, and so may have been at a low enough energy to be under the instrument detection limit. Also, due to these same effects, only two sources out of the 22 showed up in the EGRET survey. This limited the analysis of the SEDs, reducing the study from considering the double-peak structure to only focusing on where the synchrotron peak would occur for each object.

The first peak frequency vs. figure of merit graph produced showed no correlation between the factors (Figure 2). No dependence on frequency of the synchrotron peak was shown for the FoM, and the BL Lac data points and the FSRQ were not separated in any fashion on the graph. However, the appearance of the graph containing the redshiftcorrected FoM (Figure 3) suggests a correlation. Though the location of the synchrotron peak frequency does not seem to depend on the object type, the 18 data points that included redshift data appear to relate $v_{\text {peak }}$ and FoM on the log-log graph. The implications of this will be discussed below.

\section{DISCUSSION AND CONCLUSIONS}

Before this study was conducted, it was understood that the synchrotron peak could fall anywhere in the IR to soft x-ray range, depending on the type of object 
involved. Such a wide range, however, was not found for the current data. Though a peak frequency varying from IR to x-ray was expected, many sources in the group with sufficient data had a synchrotron peak in IR/near-IR. All but 3 objects of the 20 to which a curve was fitted have synchrotron peaks in this range, suggesting that they fall into the LBL and FSRQ categories.

This effect could have been due to many factors. The first, though the author is not convinced of it, would be that the blazar population in general has an $85 \%$ tendency to have the synchrotron peak in the IR range. This is highly improbable due to the current division of blazar types. One type of blazar has not excessively been found over other types in current surveys, suggesting that one category of object is not more likely to occur than another. A more reasonable scenario is that the data source selection methods are biased. Most of the figures plotted were for objects that had data in the IRAS survey, which means that they were selected to be objects that would have a detectable energy emission in the IR. Because blazars are less variable in the IR than in other wavelengths, those which are not too faint to be detected by IR instruments, or those normally peaking in the IR range, would be preferentially selected. Along the same lines, the objects that may have had a peak frequency at another wavelength may have been too faint in the IR to be detected, automatically removing them from this sample.

However, the final result of this survey was also biased in other ways. As mentioned before, a synchrotron peak frequency vs. FoM graph was created to see if there was a correlation between the two factors (Figure 2). If a trend were apparent in such a graph, it would signify that the FoM used to select the sources in the original data list biased the sample toward objects with peaks in the IR or optical wavelengths. Such an 
effect has been analyzed for the results of past surveys. For instance, a recent analysis (4: 226) suggests that the different categories of identified blazars found in each survey could be due only to selection criteria biases. As stated in that study, most LBL sources have been identified in the radio range, while HBL are most often found in X-ray surveys.

While no correlation occurs between FoM and the frequency of the synchrotron peak for BL Lacs or FSRQ in the original graph (Figure 2), the dependence of the modified FoM on synchrotron peak frequency (Figure 3) suggests that the FoM is biased to select objects peaking at lower frequencies (i.e. LBL and FSRQ). This selection effect has many different implications. The FoM in the work by Sowards-Emmerd et al. is based on radio fluxes as well as radio spectra, though one term for x-ray flux was included to create less bias. They believed in that study that the radio properties of a blazar object correspond well with the gamma variability and properties, and so it was more worthwhile to focus on that part of the broadband spectra to select blazar candidates. This decision has weighted the survey toward objects with synchrotron peaks in the radio to optical range, as can be seen on the graph. While part of the bias can be explained by the infrared selection of objects in this survey, it cannot be denied that any data input for this model would have a higher figure of merit and be more likely be selected if it were an FSRQ or LBL. Perhaps the inclusion of terms for the IR, near-IR, optical, as well as $\gamma$-ray flux in the FoM calculation would make a more broadly based model for the selection of all blazars, or different FoM systems could be determined for pinpointing the three different types. This more inclusive model would be necessary to 
continue the study of blazar properties in general, instead of focusing on such a confined sample. For now, this is can only be left up to further study.

If one examines the merits of such a limited set of objects, however, other patterns arise. Another interpretation of the corrected FoM graph would be that, depending on where the synchrotron peak occurred for a blazar candidate, a higher luminosity or intensity of peak would be expected for lower peak frequencies. This effect mirrors that found by Fossati et al. in their 1998 study (5). They discovered a correlation between the luminosity of a blazar and the synchrotron peak frequency of the type of object involved. According to their study, the intensity of the synchrotron and Compton scattering peaks in blazar SEDs are dependent on peak frequency. The linear tendency they found in their $v$ vs. $v \mathrm{~L}_{v}$ plots (5: 441) is strikingly similar to that found in Figure 3, where the luminosity is expected to be higher for lower frequency peaks. Indeed, the luminosity progression theorized by Fossati et al. can be seen in a luminosity curve plot of each object (Figure 4). As you move to greater frequencies along the plot, the maximum luminosity peak found for the objects lessens.

The objects that fall in the lower frequency range for the synchrotron peak are more likely to show a $\gamma$-ray Compton radiation peak in the $\mathrm{GeV}$ range, according to the results of the Fossati et al. study. Under this assumption, the FoM defined in the survey by Sowards-Emmerd et al. immediately becomes very important for further observational study of blazar candidates. The objects most likely to be selected by the FoM as it was originally defined would have a $\gamma$-ray peak occurring in the $\mathrm{GeV}$, the range most easily detected by the EGRET survey, and most accurately detected by the upcoming GLAST mission. The sensitivity and depth of exposure of the future satellite data will resolve the 
issues of discovering objects that are faint in the $\gamma$-ray band, as well as making it possible to study variability with its frequent viewings of each source, furthering what we already have discovered about these objects. However, until GLAST has been launched, the FoM defined in the Sowards-Emmerd et al. survey could be used to compile a more complete list of blazar candidates over the entire sky. GLAST will study these objects in more detail, but it would be worthwhile to have a ranked list ready and waiting to be examined. That is the ultimate goal of this study: providing a basis for progress in the study of blazar dynamics with a more sensitive and complete catalogue of blazar-like objects, and being prepared to use advances in technology efficiently, taking advantage of GLAST results as soon as they are available.

\section{ACKNOWLEDGMENTS}

This research was conducted at the Stanford Linear Accelerator Center. I thank the U. S. Department of Energy, Office of Science for giving me the opportunity to participate in the SULI program and the chance for me to have a rewarding learning experience. My greatest regards go to Helen Quinn and James Lindesay for running this program. This research has made use of the NASA/IPAC Extragalactic Database (NED) which is operated by the Jet Propulsion Laboratory, California Institute of Technology, under contract with the National Aeronautics and Space Administration. This study would also not have been possible without the use of NASA's Astrophysics Data System Abstract Service. Special thanks go to my mentors Greg Madejski and Seth Digel at the Stanford Linear Accelerator Center for their knowledge, insight, time and patience. I also thank David Sowards-Emmerd et al. for the contribution of data from their recent sky 
survey and the enthusiasm shown toward my project idea, Phil Marshall at SLAC for his distance program, as well as my colleagues at SLAC who gladly answered questions.

\section{WORKS CITED}

[1] G. Ghisellini et al., "A Theoretical Unifying Scheme for Gamma-ray Bright Blazars," arXiv: astro-ph/9807317v1, July 30, 1998. 1-24.

[2] David Sowards-Emmerd et al., "A Northern Survey of Gamma-Ray Blazar Candidates," Astrophysical Journal, vol. 626, 95-103, June 10, 2005.

[3] David W. Hogg, "Distance Measures in Cosmology,” arXiv: astro-ph/9905116v4, Dec.16, 2000. 1-16.

[4] S. Antón and I. W. A. Browne, "The Recognition of Blazars and the Blazar Spectral Sequence," RAS, vol. 356, 225-231, Sept. 23, 2004.

[5] G. Fossati et al., "A Unifying View of the Spectral Energy Distributions of Blazars," RAS, vol. 299, 433-448, 1998.

\section{OTHER REFERENCES}

M. Böttcher et al., "Coordinated Multiwavelength Observations of BL Lacertae in 2000," Astrophysical Journal, vol. 596, 847-859, Oct. 20, 2003.

S. Ciprini et al., "The Infrared Peak of the Blazar Spectral Energy Distribution and the Monitoring from Antarctica," Mem. S.A.It., vol. 74, 70, 2003.

Giovanni Fossati, "Demographics of Blazars," arXiv, Dec 21, 2000.

P. Giommi et al., "The Sedentary Multifrequency Survey, I. Statistical Identification and Cosmological Properties of High-Energy Peaked BL Lacs," RAS, vol. 310, 465-475, July 6, 1999.

H. Kubo et al., "ASCA Observations of Blazars and Multiband Analysis," Astrophysical Journal, vol. 504, 693-701, Sept. 10, 1998.

Greg M. Madejski, "Black Holes in Active Galactic Nuclei: Observations," Theory of Black Hole Accretion Disks, Cambridge University Press. 1-21. 
C. von Montigny et al., "High Energy Gamma-Ray Emission from Active Galaxies:

EGRET Observations and Their Implications," Astrophysical Journal, vol. 440, 525-553, Feb. 20, 1995.

Paolo Padovani and Paolo Giommi, "The Connection Between X-Ray- and RadioSelected BL Lacertae Objects," Astrophysical Journal, vol. 444, 567-581, May 10, 1995.

Marek Sikora and Greg Madejski, "Blazars," arXiv, Jan. 22, 2001. 
Tables

Table 1- Frequency used to plot each wavelength band

\begin{tabular}{|c|c|}
\hline BAND & FREQUENCY (Hz) \\
\hline Radio & $8.4 \times 10^{9}$ \\
\hline $100 \mu \mathrm{m}$ & $2.99 \times 10^{12}$ \\
\hline $60 \mu \mathrm{m}$ & $4.983 \times 10^{12}$ \\
\hline $25 \mu \mathrm{m}$ & $1.196 \times 10^{13}$ \\
\hline $12 \mu \mathrm{m}$ & $2.492 \times 10^{13}$ \\
\hline $\mathbf{J}$ & $2.411 \times 10^{14}$ \\
\hline $\mathbf{H}$ & $1.801 \times 10^{14}$ \\
\hline $\mathbf{K}_{\mathrm{s}}$ & $1.384 \times 10^{14}$ \\
\hline Red & $4.425 \times 10^{14}$ \\
\hline Visible & $6.34 \times 10^{14}$ \\
\hline X-ray & $2.524 \times 10^{17}$ \\
\hline$\gamma$-ray & $\begin{array}{c}\text { Individually determined }<v>\text { for each } \\
\text { object }\end{array}$ \\
\hline
\end{tabular}

Table 2- Pertinent data for the FoM graphs and scaled FoM calculations

\begin{tabular}{|c|c|c|c|c|c|c|c|c|c|}
\hline Object Name & $\begin{array}{l}\text { FoM } \\
\text { (S-E, } \\
2005)\end{array}$ & $\begin{array}{c}\mathrm{S}_{8.4} \\
(\mathrm{mJy})\end{array}$ & $\alpha$ & $\begin{array}{l}\text { RASS } \\
\text { count } \\
(\mathbf{c t} / \mathbf{s})\end{array}$ & $\mathbf{z}$ & Type & $\begin{array}{l}\text { Voeak }_{\text {peak }} \\
(\mathbf{H z})\end{array}$ & $\begin{array}{l}D_{L} \\
\left(D_{\text {med }}=\right. \\
1122.9 \\
\text { Mpc) }\end{array}$ & $\begin{array}{c}\text { Scaled } \\
\text { FoM }\end{array}$ \\
\hline J003939.61+141157.5 & .075 & 369.8 & -0.19 & 0 & 1.738 & B & $2.91 \times 10^{12}$ & 6409.262 & 0.112 \\
\hline J011205.82+224438.7 & .105 & 493.4 & -0.15 & 0.151 & ----- & $\mathbf{B}$ & $1.02 \times 10^{14}$ & ----- & ----- \\
\hline J024918.01+061951.9 & .079 & 579.3 & -0.1 & $\mathbf{0}$ & 1.881 & $\mathbf{F}$ & $4.88 \times 10^{12}$ & 7062.936 & 0.084 \\
\hline J025134.53+431515.8 & .079 & 1174.1 & 0.01 & 0.027 & 1.31 & $\mathbf{F}$ & $2.68 \times 10^{13}$ & 4521.92 & 0.124 \\
\hline J030335.24+471616.2 & .132 & 1616.3 & -0.29 & $\mathbf{0}$ & .475 & B & $5.29 \times 10^{12}$ & 1305.421 & 0.136 \\
\hline J031948.16+413042.1 & .092 & 34296 & -0.23 & $\mathbf{0}$ & .017 & $\mathbf{F}$ & $5.02 \times 10^{13}$ & 36.142 & -0.069 \\
\hline J040922.00+121739.8 & .103 & 667.3 & -0.25 & $\mathbf{0}$ & 1.02 & B & $3.62 \times 10^{12}$ & 3317.977 & 0.131 \\
\hline J043311.09+052115.6 & .088 & 2105.2 & 0.26 & 1.705 & .033 & $\mathbf{F}$ & $2.03 \times 10^{14}$ & 71.002 & -0.072 \\
\hline J050925.96+054135.3 & .093 & 702.4 & -0.16 & 0.042 & $\begin{array}{ll}---- \\
\end{array}$ & $\mathbf{B}$ & $3.75 \times 10^{13}$ & ----- & ----- \\
\hline J075706.64+095634.8 & .143 & 1363.5 & -0.23 & 0.113 & .66 & $\mathbf{B}$ & $7.41 \times 10^{13}$ & 1942.478 & 0.206 \\
\hline J082550.33+030924.5 & .145 & 1873.4 & -0.18 & 0.132 & .506 & $\mathbf{B}$ & $3.41 \times 10^{13}$ & 1408.145 & 0.169 \\
\hline $\mathrm{J} 140700.39+282714.6$ & .167 & 1936.3 & -0.49 & $\mathbf{0}$ & .076 & $\mathbf{F}$ & $7.26 \times 10^{13}$ & 168.631 & -0.067 \\
\hline J141558.81+132023.7 & .119 & 1564.3 & -0.21 & $\mathbf{0}$ & .246 & B & $3.06 \times 10^{12}$ & 606.272 & 0.086 \\
\hline J141946.59+542314.7 & .219 & 2248.1 & -0.62 & 0.103 & .1153 & $\mathbf{B}$ & $3.20 \times 10^{13}$ & 357.109 & 0.084 \\
\hline J155543.04+111124.3 & .195 & 506.5 & -0.28 & 1.513 & .36 & B & $3.99 \times 10^{14}$ & 940.368 & 0.165 \\
\hline J164258.80+394836.9 & .126 & 6299.8 & 0.05 & 0.325 & .593 & $\mathbf{F}$ & $2.47 \times 10^{13}$ & 1705.328 & 0.134 \\
\hline J180045.68+782804.0 & .117 & 2874 & -0.15 & 0.066 & .68 & $\mathbf{F}$ & $3.11 \times 10^{13}$ & 2014.571 & 0.158 \\
\hline J180650.68+694928.1 & .11 & 1595.5 & $\mathbf{0}$ & 0.154 & .05 & $\mathbf{B}$ & $1.09 \times 10^{14}$ & 108.921 & -0.100 \\
\hline J195959.84+650854.6 & .071 & 222.8 & 0.04 & 2.653 & .048 & B & $1.12 \times 10^{15}$ & 104.413 & -0.244 \\
\hline J221949.74+261327.9 & .115 & 427 & -0.41 & 0.085 & .085 & $\mathbf{F}$ & $7.91 \times 10^{13}$ & 189.774 & -0.203 \\
\hline
\end{tabular}


Table 3- Statistics on sources from which data was combined.

\begin{tabular}{|c|c|c|c|}
\hline Mission & IRAS & 2MASS & EGRET \\
\hline $\begin{array}{c}\text { Wavelengths/ } \\
\text { energy band }\end{array}$ & $\begin{array}{c}\text { IR- 12, 25, 60, 100 } \\
\text { microns }\end{array}$ & $\begin{array}{c}\text { Near-IR- 1.24, } \\
1.66,2.16 \text { microns }\end{array}$ & $\begin{array}{c}\gamma \text {-ray- 20 MeV-30 } \\
\text { GeV }\end{array}$ \\
\hline $\begin{array}{c}\text { Effective } \\
\text { Minimum } \\
\text { Energy/Flux }\end{array}$ & $\begin{array}{c}\text {.5 Jy at } 12 \text { microns } \\
\text { to } 1.5 \mathrm{Jy} \text { at } 100 \\
\text { microns }\end{array}$ & $1 \mathrm{mJy}$ & $100 \mathrm{MeV}$ \\
\hline Positional accuracy & $\mathbf{2 0 ”}$ & $\mathbf{. 5}$ & $\mathbf{1 0 \%}$ \\
\hline Sky Coverage & $\mathbf{9 6 \%}$ & $\sim 95 \%$ & $\sim 99 \%$ \\
\hline
\end{tabular}


Figures
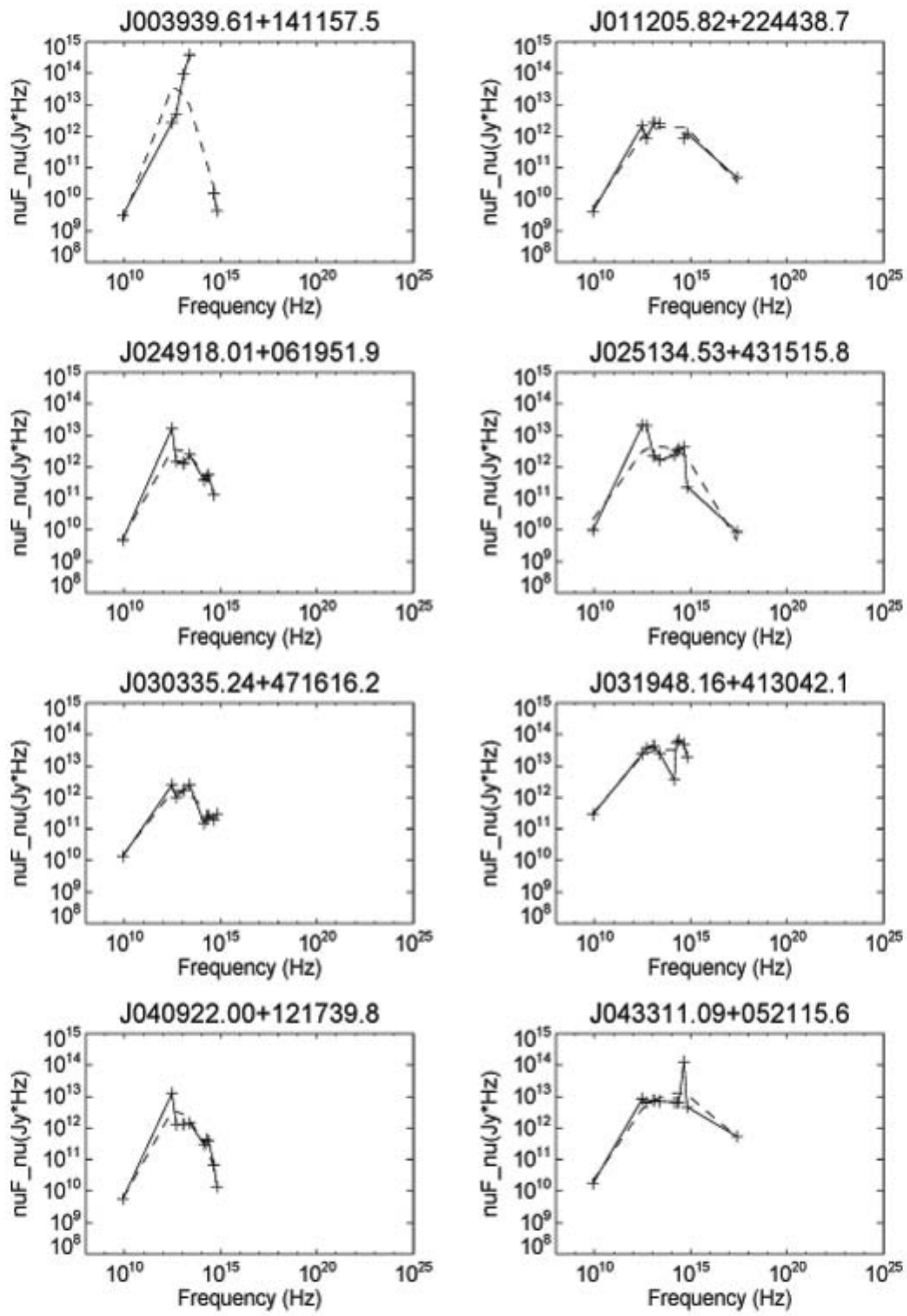

Figure 1- Plotted SEDs of 22 objects with adequate data. Solid lines connect the observed data, dashed lines model the computer-fitted curve. 

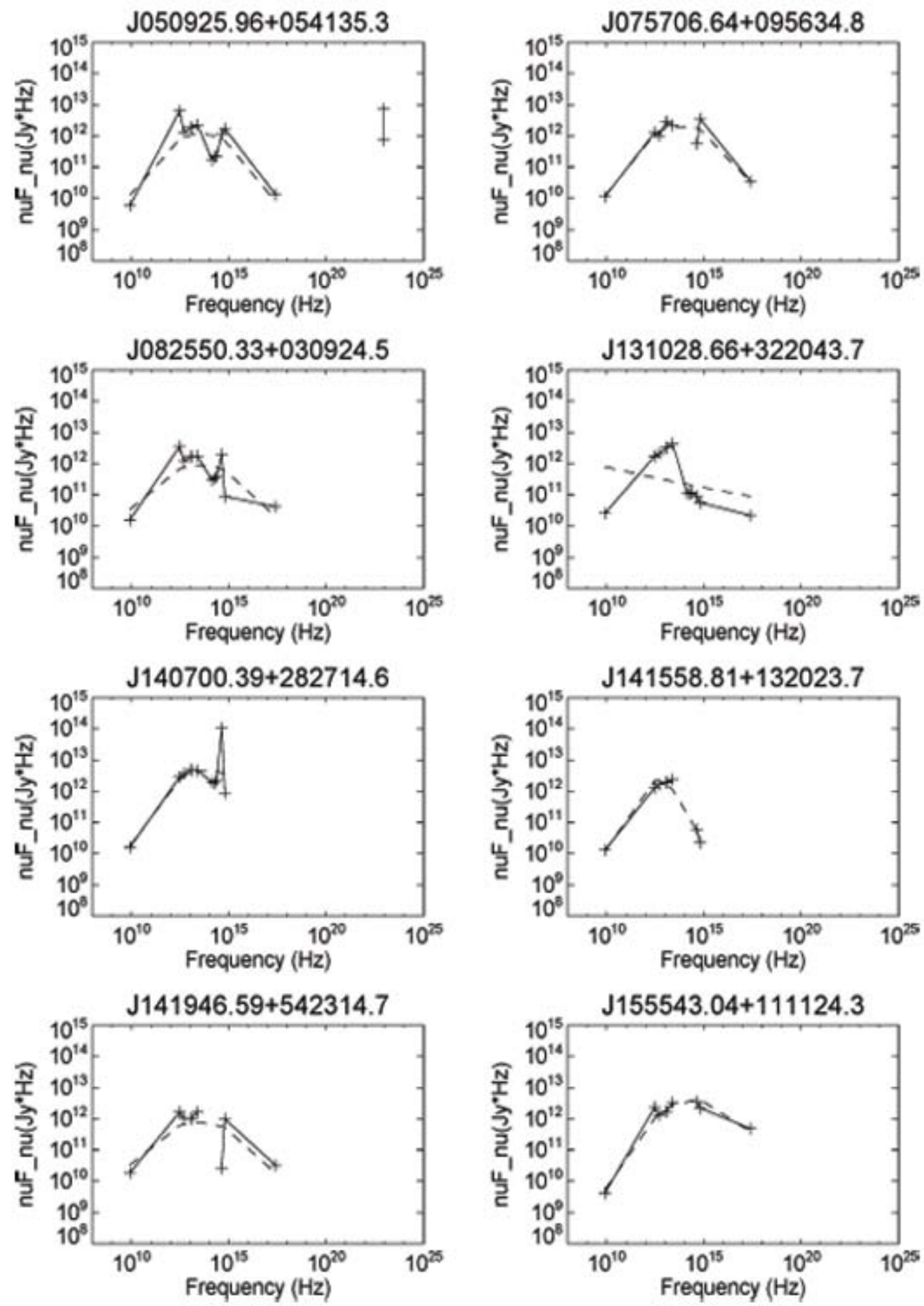

Figure 1- continued 

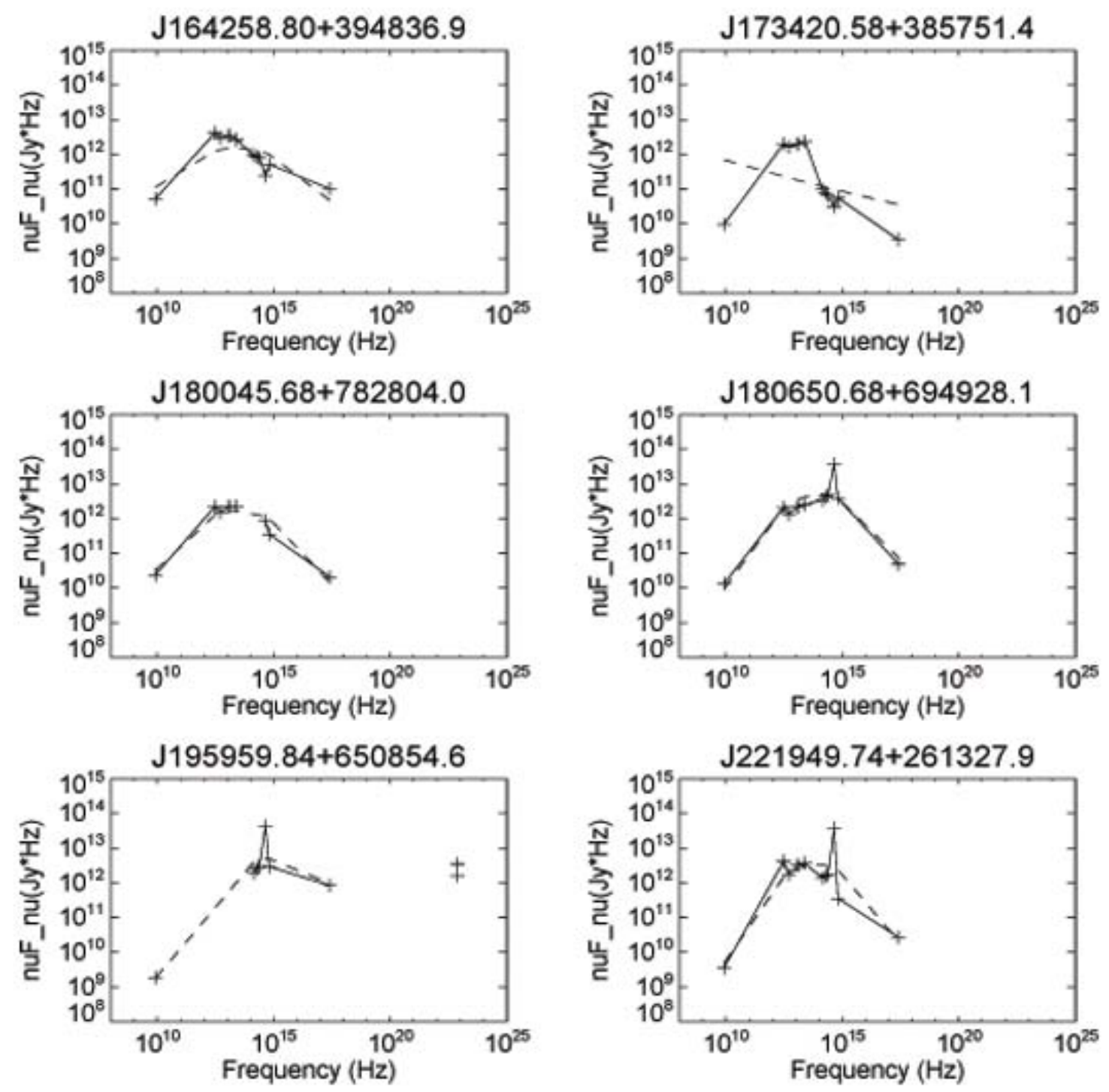

Figure 1- continued

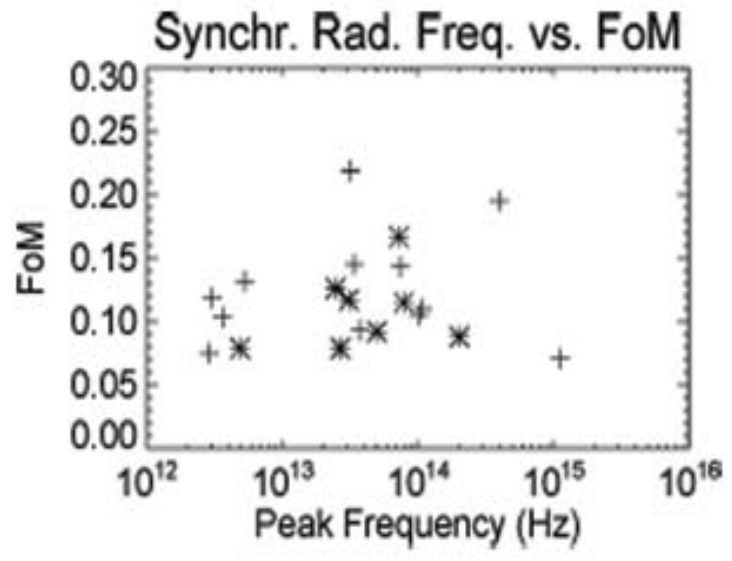

Figure 2- BL Lac plotted as +, FSRQ plotted as * 


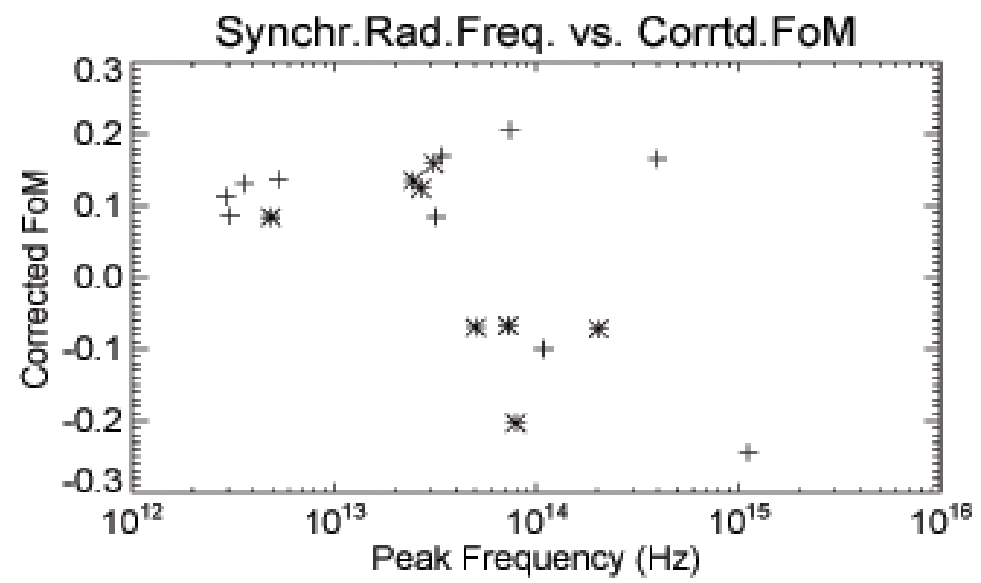

Figure 3- FoM corrected for redshift. As above (Figure 2), BL Lac plotted as +, FSRQ plotted as *. Only objects with a defined redshift were included in this graph.

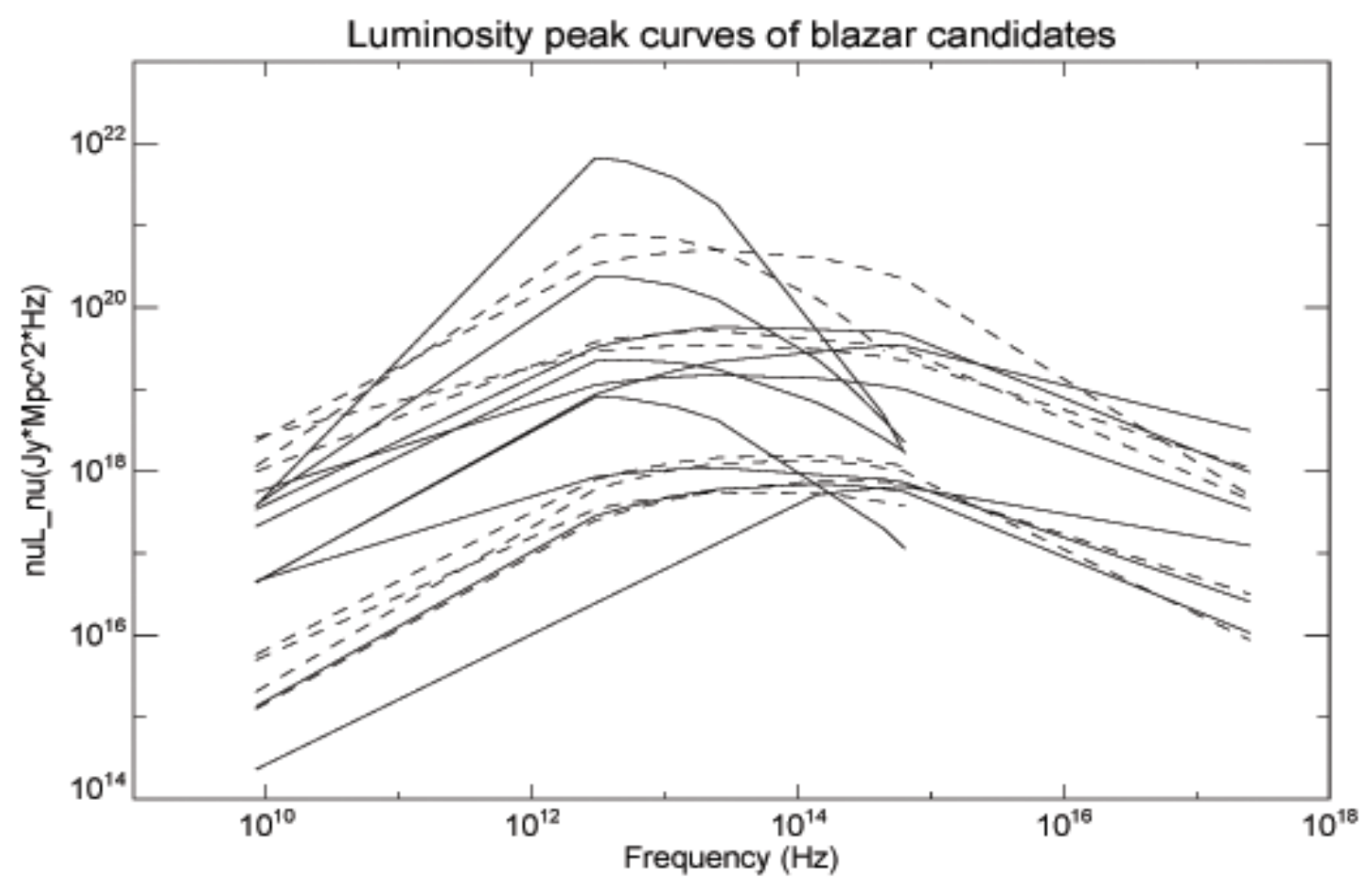

Figure 4- Synchrotron luminosity curves for the 18 redshift-correctable objects, illustrating luminosity progression theory. Parabola curves were fitted to the luminosity data in the same manner as the SED graphs, creating the peaks for each object seen in the compilation plot above. 